\title{
Optical/digital incoherent image processing for extended depth of field
}

\author{
Ting-Chung Poon and Masoud Motamedi
}

\begin{abstract}
For a severely defocused incoherent system, its optical transfer function (OTF) has isolated zeros; therefore, an exact inverse filtering cannot be performed. Isolated zeros in the OTF can be avoided by choosing an annular aperture with a proper radius ratio, as the aperture can extend the depth of focus of the system. However, in the process of increasing the depth of focus of the system, this method results in a loss of image contrast. A simple hybrid optical/digital image processing system in which a TV camera device is coupled with an annular aperture is considered. Annular-pass filtering to compensate for the loss of contrast is performed by a digital computer. Experimental results are presented.
\end{abstract}

\section{Introduction}

Throughout the years, different methods have been considered to reduce the amount of misfocus. The effect of graded apertures on a defocused system has been studied. ${ }^{1}$ The use of Fresnel zone plates has been shown to improve the optical transfer function (OTF) and leads to a larger depth of field. ${ }^{2}$ A number of methods have been proposed for digitally analyzing a sequence of images, each of which is composed of infocus as well as out-of-focus regions. ${ }^{3-5}$ In the context of hybrid image processing to increase the depth of field, a two-step method has been introduced. ${ }^{6}$ In the first step of this method, the object is moved along the optical axis through a distance somewhat longer than the object thickness to obtain the integrated image. The integration would smooth the OTF of the system, thereby getting rid of the zeros of the out-of-focus transfer function. Subsequent conventional coherent image processing compensates for the attenuation of high frequency components due to the first step. In another hybrid method, ${ }^{7}$ the object is vibrated rapidly in front of a vidicon camera, and the video output is highpass filtered by the use of electronic circuitry.

When this work was done both authors were with Virginia Polytechnic Institute \& State University, Department of Electrical Engineering, Blacksburg, Virginia 24061; M. Motamedi is now with Geostar Corporation, 1001 22nd Street NW, Washington, DC 20037.

Received 26 March 1987.

0003-6935/87/214612-04\$02.00/0.

(C) 1987 Optical Society of America.
It has been established that, by using an annular aperture in an optical system, the depth of focus can be increased. ${ }^{8}$ However, the use of annular apertures has not received much practical consideration in the past. The first practical hybrid system using annular apertures of high aspect ratios and postcoherent image processing to increase the depth of field was considered by McCrickerd. ${ }^{9}$ Annular apodizers have recently been studied. ${ }^{10}$ In this paper, a hybrid optical/ digital incoherent image processing system is considered in which a TV camera device coupled with an annular aperture is used. Isolated zeros in the OTF of a defocused system are avoided by choosing an annular aperture with a proper radius ratio. The loss of image contrast is restored by using a computer-generated annular-pass filter. In the next section, the theory behind the increase of the depth of focus by using the annular aperture is discussed, and its effect on the contrast of the image and the method to compensate for it are explained. In the last two sections, we present some experimental results and make some concluding remarks.

\section{Annular Aperture: Depth of Focus, Optical Transfer Function, Inverse Filtering}

\section{A. Depth of Focus}

An annular aperture is defined as a clear circular pupil with a central obstruction. If the aperture is an annulus with outer radius $a$ and inner radius $b$, we can define a central obscuration ratio of $\epsilon=b / a$. Consider the lens system shown in Fig. 1. We want to find an expression for the depth of focus $\delta z$ in terms of the known quantities of the system or quantities that can easily be measured. A simple quantum mechanical argument is convenient to use here. ${ }^{11}$ Quantum me- 
chanics relates the minimum uncertainty in position of a quantum $\delta z$ to the uncertainty in its momentum $\delta p_{z}$ according to

$$
\delta z \delta p_{z} \cong h,
$$

where $h$ is Planck's constant and $p_{z}$ is the $z$ component of the momentum. For the lens system with a clear aperture with radius $a$, shown in Fig. 1 (a), $\delta p_{z}$ signifies the momentum difference between rays $A A^{\prime}$ and $O O^{\prime}$ and is given by

$$
\delta p_{z}=p_{0}-p_{0} \cos (\beta / 2),
$$

where $p_{0}=h / \lambda$ is the momentum of the quantum. Substituting Eq. (2) into Eq. (1), we have

$$
\delta z=\lambda /[1-\cos (\beta / 2)],
$$

which can be written as

$$
\begin{aligned}
\delta z & =\lambda /\left[1-\sqrt{1-\sin ^{2}(\beta / 2)}\right] \\
& \cong 2 \lambda / \sin ^{2}(\beta / 2),
\end{aligned}
$$

where we have used small angle approximations to obtain the last expression. Since $\beta \cong 2 a / f$, where $f / 2 a$ is the $f$ No. of the system, Eq. (4) can be shown to be approximated by

$$
\delta z \cong 8 \lambda f_{\#}^{2},
$$

where Eq. (5) is the well-known depth of focus expression for a clear aperture lens system.

We can apply the same principle to derive the depth of focus of an annular aperture. Referring to Fig. 1(b), $\delta p_{z}$ in this case is the momentum difference between the ray passing the upper part of the aperture and the ray passing the lower part of the aperture and is given by

$$
\delta p_{z}=\delta p_{a}-\delta p_{b},
$$

where $\delta p_{a}$ and $\delta p_{b}$ are given by Eq. (2) with $\beta$ and $\alpha$ substituted into the argument of cosine, respectively. Hence Eq. (6) becomes

$$
\delta p_{z}=p_{0}[\cos (\alpha / 2)-\cos (\beta / 2)],
$$

and the depth of focus is given by

$$
\delta z=\lambda /[\cos (\alpha / 2)-\cos (\beta / 2)],
$$

which can be shown to have the form

$$
\delta z=\frac{\lambda}{\sqrt{1+\left(\frac{\epsilon}{2 f_{\#}}\right)^{2}}}-\frac{1}{\sqrt{1+\left(\frac{1}{2 f_{\#}}\right)^{2}}},
$$

and assuming $f_{\#} \gg \epsilon$, Eq. (9) can be approximated by

$$
\delta z \cong 8 \lambda f_{\#}^{2} /\left(1-\epsilon^{2}\right) .
$$

The numerator of (10) is the same as (5) derived for the depth of focus of the single lens with no annular aperture. Therefore, the depth of focus of a system with a central obstruction is greater than that of the system without the obstruction by a factor of $1 /\left(1-\epsilon^{2}\right)$. Linfoot and Wolf have showed the same expression for the increase of the depth of focus. ${ }^{12}$ With $95 \%$ obstruc-

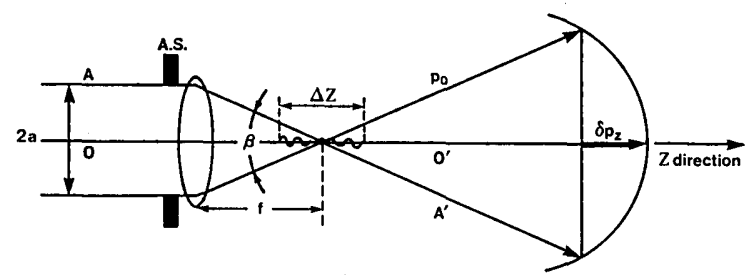

(a)

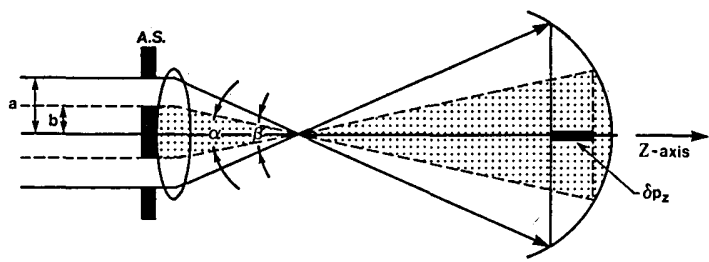

(b)

Fig. 1. Uncertainty principle used in finding depth of focus: (a) lens with no central obstruction; (b) lens with annular aperture.

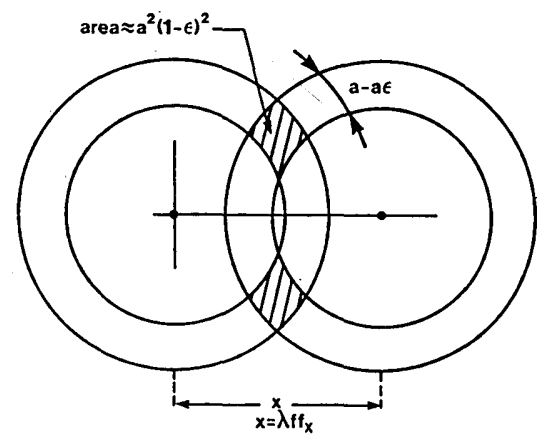

Fig. 2. Autocorrelation of annular pupil function.

tion, i.e., $\epsilon=0.95$, we can increase the depth of focus of a clear lens by more than a factor of 10 . It should be mentioned that as $\epsilon \rightarrow 1$ lateral resolution does not suffer while increasing the depth of focus in an annular system, as the spread in momentum along the lateral dimensions is the same as that in an unobstructed aperture system. ${ }^{13,14}$

\section{B. Optical Transfer Function}

The optical transfer function (OTF) is given by the autocorrelation of the aperture function. Correlation of annular aperture function in one dimension can be visualized by considering Fig. 2. The displacement between the center of the pupil and the center of its complex conjugate is denoted $X=\lambda f f_{x}$. Originally, when the two centers are overlapped, it gives the maximum response at zero frequency:

$$
\begin{aligned}
\operatorname{OTF}(0) & =\pi\left(a^{2}-b^{2}\right) \\
& =\pi a^{2}\left(1-\epsilon^{2}\right) .
\end{aligned}
$$

This value decreases monotonically for $0<f_{x}<a(1-$ $\epsilon) / \lambda f$. The correlation value then remains about at the value $2 a^{2}(1-\epsilon)^{2}$ for $a(1-\epsilon) / \lambda f<f_{x}<2 a / \lambda f$. The approximate cross section along $f_{x}$ of the OTF is sketched in Fig. 3(a), and the normalized OTF is illustrated in Fig. 3(b). An exact transfer function for an 


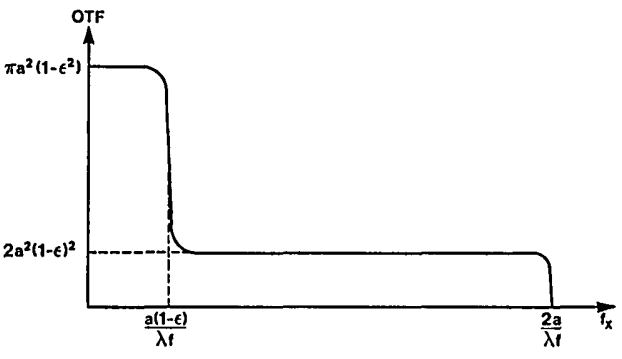

(a)

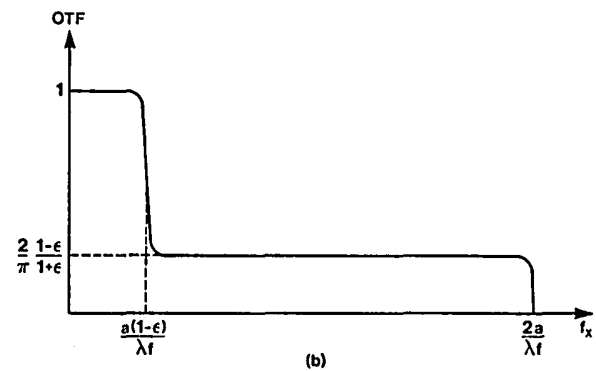

Fig. 3. (a) OTF of system with annular aperture; (b) normalized OTF.

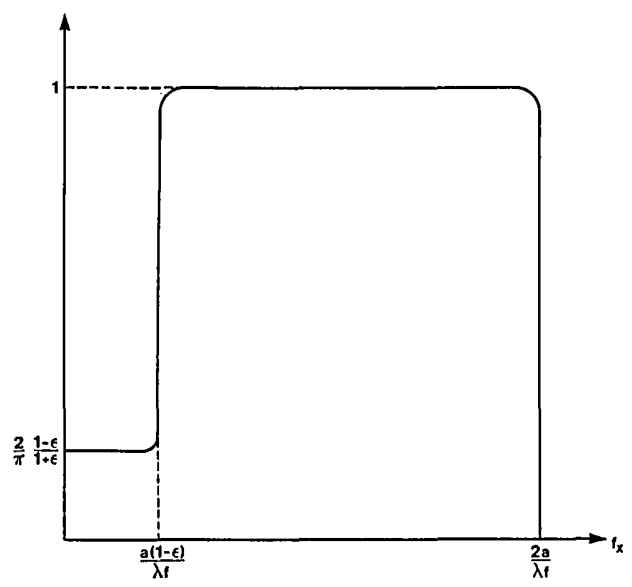

Fig. 4. Annular-pass filter.

annular aperture has been given by O'Neill. ${ }^{15}$ We can readily see that such a system will largely attenuate the high frequency components of the image while passing the low frequency components (including dc) essentially unattenuated. Thus the detailed information of the image is embedded in a strong low frequency background.

\section{Inverse Filtering}

From the discussion of the last two sections, we can see that by using an annular aperture in an optical system, the depth of focus can be increased. By properly choosing the radius ratio of the annular aperture, we can entirely eliminate the zeros in the OTF of a defocused system. However, in the process of increasing the depth of focus, this method results in a loss of image contrast. Now, suppose we have an object of which the spectrum $F_{d} F_{i}$ represents a picture taken

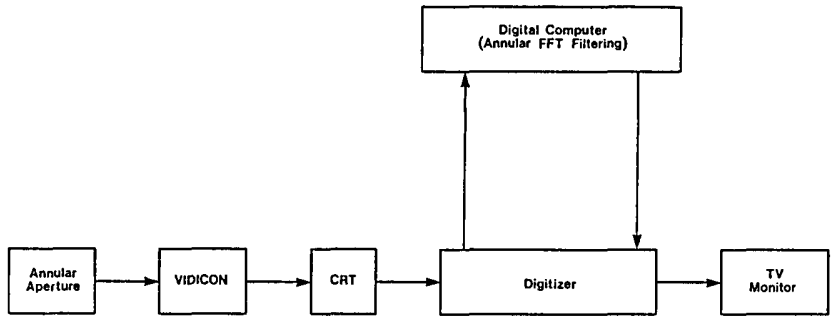

Fig. 5. Optical/digital image processing system.

with a lens and an annular aperture, where $F_{i}$ denotes the ideal image without degradation and $F_{d}$ a filter function which expresses the degradation and is shown in Fig. 3(b). We can restore $F_{i}$ by using a filter with the transfer characteristic $F_{d}^{-1}$. In this specific case, $F_{d}^{-1}$ must have the characteristics as shown in Fig. 4. In two dimensions, this is an annular-pass filter.

\section{Hybrid Processing System and Experimental Results}

The combination of the optical system, digital system, and their interface, as applied to image processing with an extended depth of field, is shown in Fig. 5. The digital system is a VAX-11/780 computer capable of running image processing software. Inverse filtering is done by the digital system. The digital processing of the image begins after insertion of the annular aperture. The process involves taking the FFT of the image and then applying the annular-pass filtering on it. The filtered results are then taken through an inverse FFT, and the final output is displayed. Figure 6(a) shows an in-focus text "Virginia Tech". The outof-focus text, as shown in Fig. 6(b), is then processed using an annular aperture, which resulted in the image shown in Fig. 6(c). An annulus of $\epsilon=0.47$ is used to bring the text back into focus, and the lens is an 8-mm movie lens with a focal length of $75 \mathrm{~mm}$. Figure 6(d) shows the digitally filtered image. The restored image exhibits some edge enhancement, which might be due to an overcompensation by the approximate annularpass filtering. Exact annular-pass filtering can only be performed if the exact OTF of the whole system is known.

\section{Concluding Remarks}

We have presented and demonstrated the feasibility of extending the depth of field of an incoherent imaging system through a hybrid (optical/digital) technique. An annular aperture is put in front of a TV camera to increase the depth of field of the system. The loss of image contrast due to the aperture is compensated by the use of approximate inverse filtering in a digital computer. It should be noted that since the OTF contains no zeros, an exact inverse filtering can be performed in principle, whereby precise OTF measurements of the system have to be done. If the restored image is obviously disturbed by noise, we can take advantage of the computer to improve the SNR in the restored image by carefully tuning the inverse filtering, i.e., deliberately altering the numerical values 


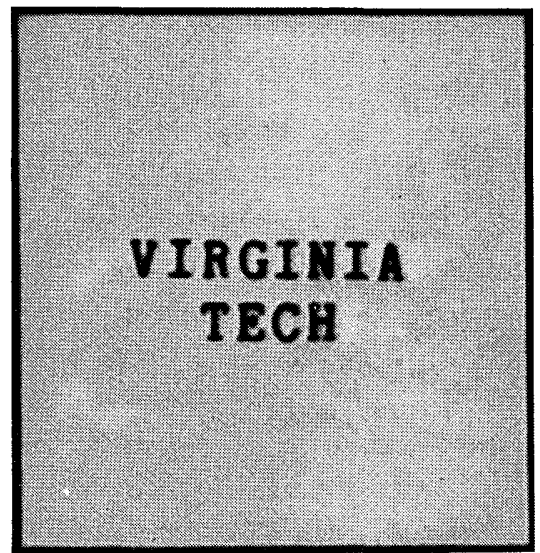

(a)

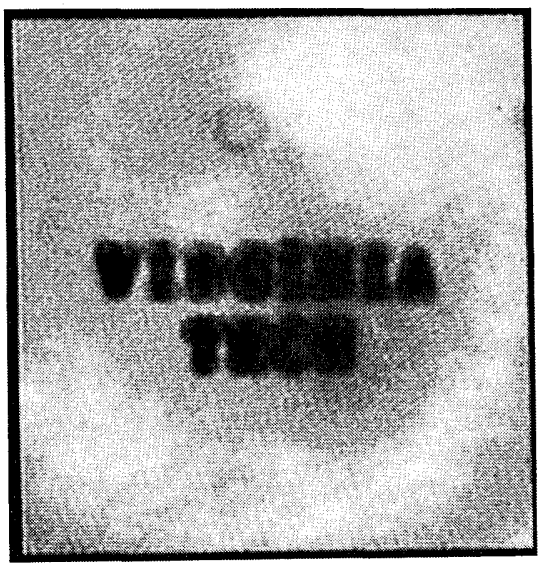

(c)

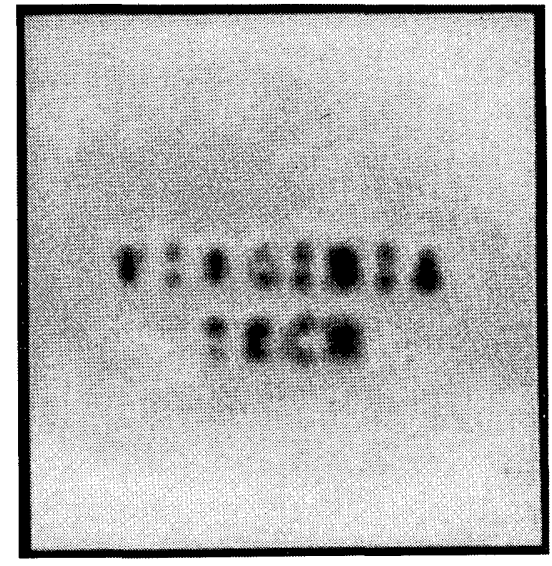

(b)

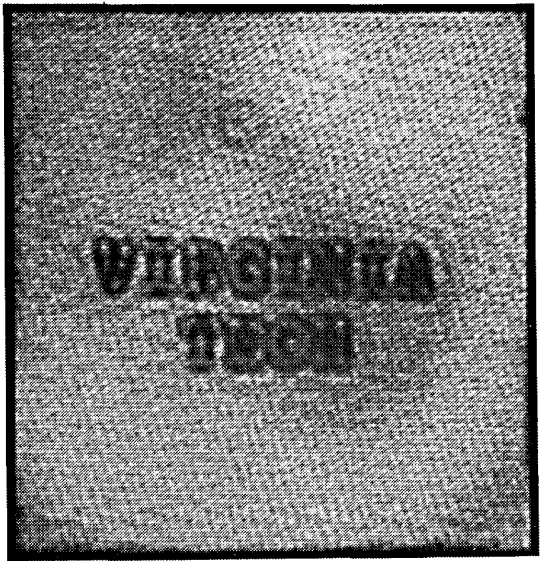

(d)

Fig. 6. (a) In-focus text; (b) severely out-of-focus text; (c) processing of (b) using annular aperture; (d) annular-pass filtered output of (c).

of the filtering. Alternatively, Wiener filtering could be employed.

The authors wish to acknowledge discussions with A. Korpel, G. Indebetouw, and K.-B. Yu. M. Motamedi also expresses his thanks to G. Bushman, G. Fabregas, and B. Stafford for assistance in using the equipment at the Spatial Data Analysis Laboratory. Finally, T.-C. Poon would like to acknowledge the support of the Fiber and Electro-Optics Research Center at Virginia Tech that made this paper possible.

\section{References}

1. M. Mino and Y. Okano, "Improvement in the OTF of a Defocused Optical System Through the use of Shaded Apertures," Appl. Opt. 10, 2219 (1971).

2. G. Indebetouw and H. Bai, "Imaging with Fresnel Zone Pupil Mask: Extended Depth of Field," Appl. Opt. 23, 4299 (1984).

3. R. J. Pieper and A. Korpel, "Image Processing for Extended Depth of Field," Appl. Opt. 22, 1449 (1983).

4. S. A. Sugimoto and Y. Ichioka, "Digital Composition of Images with Increased Depth of Focus Considering Depth Information," Appl. Opt. 24, 2076 (1985).

5. G. Häusler and E. Körner, "Expansion of Depth of Focus by Image De-Puzzling," in Proceedings, Sixth International Conference on Pattern Recognition (1982), p. 1201.
6. G. Hausler, "A Method to Increase the Depth of Focus by Two Step Image Processing," Opt. Commun. 6, 38 (1972).

7. G. Häusler and A. Lohmann, "Hybrid Image Processing," in Applications of Holography and Optical Data Processing, E. Maron, A. Friesem, and E. Wiener-Avnear, Eds. (Pergamon, London, 1977), p. 9.

8. W. T. Welford, "Use of Annular Apertures to Increase Focal Depth," J. Opt. Soc. Am. 50, 749 (1960).

9. J. T. McCrickerd, "Coherent Processing and Depth of Focus of Annular Aperture Imagery,” Appl. Opt. 10, 2226 (1971).

10. J. Ojeda-Castaneda, P. Andres, and A. Diaz, "Annular Apodizers for Low Sensitivity to Defocus and to Spherical Aberration," Opt. Lett. 11, 487 (1986).

11. A. Korpel, U. Iowa; private communication.

12. E. H. Linfoot and E. Wolf, "Diffraction Images in Systems with an Annular Aperture," Proc. Phys. Soc. London Ser. B 66, 145 (1953).

13. A. Korpel, "Comments on 'Diffraction-Free Beams'," submitted to Phys. Rev. Lett.

14. R. J. Pieper, J. Park, and T.-C. Poon, "Resolution-Dependent Depth of Focus for an Incoherent Imaging System," submitted to Appl. Opt.

15. E. L. O'Neill, "Transfer Function for an Annular Aperture," J. Opt. Soc. Am. 46, 285 (1956). 